\title{
Limiting Pseudomonas aenuginosa Biofilm Formation Using Cold Atmospheric Pressure Plasma
}

\author{
Bethany L. Patenall, ${ }^{a}$ Hollie Hathaway, ${ }^{b}$ Adam C. Sedgwick, ${ }^{a}$ Naing T. Thet, ${ }^{a}$ \\ George T. Williams, ${ }^{a}$ Amber E. Young, ${ }^{c}$ Sarah L. Allinson, ${ }^{d}$ Robert D. Short, ${ }^{b}$ \\ \& A. Toby A. Jenkins ${ }^{a, *}$ \\ aDepartment of Chemistry, University of Bath, UK; ' bepartment of Chemistry, Lancaster Univer- \\ sity, UK; ' The Scar Free Foundation Centre for Children's Burns Research, The Bristol Royal \\ Hospital for Children, Bristol, UK; 'Division of Biomedical and Life Sciences, Lancaster University, \\ Lancaster, UK \\ *Address all correspondence to: A. Toby A. Jenkins, Department of Chemistry, University of Bath, UK; \\ Tel.: +441225386188; Fax: +44122538623, E-mail: A.T.A.Jenkins@bath.ac.uk
}

\begin{abstract}
We investigate the ability to disrupt and limit growth biofilms of Pseudomonas aeruginosa using application of cold atmospheric pressure (CAP) plasma. The effect of the biofilm's exposure to a helium (CAP) jet was assessed at varying time points during biofilm maturation. Results showed that the amount of time during biofilm growth that CAP pressure was applied has a crucial role on the ability of biofilms to mature and recover after CAP exposure. Intervention during the early stages of biofilm formation $(0-8 \mathrm{~h})$ results in a $4-5-\log$ reduction in viable bacterial cells (measured at $24 \mathrm{~h}$ of incubation) relative to untreated biofilms. However, CAP treatment of biofilm at $12 \mathrm{~h}$ and above only results in a 2-log reduction in viable cells. This has potentially important implications for future clinical application of CAP to treat infected wounds.
\end{abstract}

KEY WORDS: plasma, biofilm, Pseudomonas aeruginosa

\section{INTRODUCTION}

Owing to the increasing disparity between the rate of antimicrobial resistance and discovery of new antibiotics, interest has grown for the use of novel antimicrobial technologies. One such field of research surrounds the use of cold atmospheric pressure (CAP) plasma, often referred to as plasma medicine. CAP therapy has proven itself to be a promising alternative to traditional antimicrobial therapies, demonstrating its ability to inactivate a wide range of pathogens, including significantly drug-resistant isolates termed ESKAPE pathogens. ${ }^{1,2}$ CAP therapy relies on delivery of a range of reactive oxygen and nitrogen species (RONS), including longer-lived species such as hydrogen peroxide $\left(\mathrm{H}_{2} \mathrm{O}_{2}\right) .{ }^{3-5}$ Already well documented are the effects of plasma-generated reactive species, including the ability to control both composition and delivery of such species according to the plasma parameters used..$^{6-9}$ As such, the versatility of CAP therapy has facilitated its use in a wide range of applications, including surface decontamination (both biotic and abiotic), equipment sterilization, microbial and spore inactivation, and cancer therapy.$^{10} \mathrm{Of}$ particular relevance to this study is the application of CAP to wound healing. In addition to its proven antimicrobial effects, studies have shown that CAP 
therapy may further enhance wound healing (at appropriate doses) via stimulation of fibroblast/keratinocyte proliferation and migration or by its proangiogenic effects, thus making it an attractive alternative treatment option for wound infection. ${ }^{8-12}$

It is estimated that between $65 \%$ and $80 \%$ of all wound infections are biofilm associated. ${ }^{13}$ Biofilm occurs when "free-living" planktonic cells adhere to a surface to form a dense community of biologically active, surface-bound microbes. Such bacterial communities are frequently encased in a polymeric layer comprised of proteins, glycoproteins, and polysaccharides, collectively known as the extracellular polysaccharide (EPS) matrix. ${ }^{14}$ In addition to the protective nature of the EPS, it also confines the cells in close proximity to each other, facilitating the activation of quorum sensing networks via the secretion of specific signaling molecules. The subsequent alteration in gene expression may control production of extracellular virulence factors and regulate specific intracellular metabolic functions, both of which contribute to the enhanced resistance of biofilms to many forms of antibiotics. ${ }^{15,16}$ Indeed, biofilm formation can increase the concentration of antimicrobial that is required by 100-1000 times, relative to planktonic cells. ${ }^{17}$

Pseudomonas aeruginosa is an opportunistic, Gram-negative bacterium that is responsible for $85 \%$ of all nosocomial infections. It is particularly prevalent in burns, causing $57 \%$ of all infections, and in cystic fibrosis, causing $30 \%$ patient mortality in ventilator-associated pneumonia. ${ }^{18,19} P$. aeruginosa uses multiple antimicrobial-resistance strategies (e.g., efflux pump-mediated resistance), exhibiting the highest levels of resistance to fluoroquinolones, ranging from $20 \%$ to $35 \%$, and increasing each year according to epidemiological trends.$^{20}$ As a result of the increasing prevalence of biofilmassociated infection, there is a growing requirement within the scientific and medical community for the development of therapeutic treatment strategies aimed at limiting and ultimately eradicating bacterial biofilms. An important consideration in the development of such technologies surrounds the recalcitrant nature of many antimicrobials toward biofilms when compared to planktonic cells. This study reports on the ability of CAP treatment to effectively reduce formation of $P$. aeruginosa biofilms, potentially increasing susceptibility to conventional treatment strategies (such as antibiotics), which, if used in conjunction, may facilitate total infection clearance.

\section{MATERIALS AND METHODS}

\section{A. Materials}

We obtained $P$. aeruginosa strain PA01 from a strain collection belonging to the Biophysical Chemistry Research Group at the University of Bath, UK. Lysogeny broth (LB), LB agar, brain-heart infusion (BHI) agar, fetal calf serum (HyClone; GE Life Sciences; Pittsburgh, PA), and LIVE/DEAD ${ }^{\mathrm{TM}}$ BacLight ${ }^{\mathrm{TM}}$ bacterial viability kits were all purchased from ThermoFisher Scientific (Loughborough, UK). The polycarbonate membranes (19-mm diameter and $0.22-\mathrm{nm}$ pore size) that we used to cultivate biofilms were purchased from Whatman (Kent, UK). Phosphate-buffered saline 
(PBS), sodium chloride $(\mathrm{NaCl})$, and peptone were all purchased from Sigma-Aldrich (Dorset, UK).

\section{B. Bacteria and Growth Conditions}

P. aeruginosa PA01 was taken from freezer stocks and grown on LB agar overnight at $37^{\circ} \mathrm{C}$ to obtain single colonies. Bacteria cultures were grown from single colonies overnight at $37^{\circ} \mathrm{C}$ with agitation $(200 \mathrm{rpm})$ in $\mathrm{LB}$, resulting in $10^{9}$ colony forming units (CFU) per milliliter in final culture. Bacterial aliquots were stored at $-80^{\circ} \mathrm{C}$ in $\mathrm{LB}$ supplemented with $15 \%(\mathrm{v} / \mathrm{v})$ glycerol.

\section{Bacterial Biofilm Formation}

Polycarbonate membranes were positioned on BHI agar and sterilized with ultraviolet light for $10 \mathrm{~min}$. We aliquoted $20 \mu \mathrm{L}$ of wound fluid mimic (fetal calf serum mixed in equal volume with $0.85 \% \mathrm{NaCl}[\mathrm{w} / \mathrm{v}]$ and $0.1 \%$ peptone $[\mathrm{w} / \mathrm{v}])$ onto membrane surfaces. Artificial wound fluid was added to the membranes before bacterial inoculation to more closely mimic the wound environment. The membranes were inoculated with $30 \mu \mathrm{L}$ of overnight bacterial culture, diluted 1:1000 into fresh LB broth. Membranes were incubated statically for $24 \mathrm{~h}$ at $37^{\circ} \mathrm{C}$. Following treatment and incubation, biofilms were stripped from the membranes into sterile PBS via sonication $(2 \times$ 15 min with 1 min vortex before and between sonication steps). The value of CFU/ $\mathrm{mL}$ was then determined via serial dilution into sterile PBS and plating on LB agar to colony count. ${ }^{21}$

\section{Plasma Treatment}

The plasma source used in this study was a helium-driven plasma jet, as previously described. ${ }^{22}$ Gas flow was fixed at 2 standard liters per min, and plasma was operated at $10 \mathrm{kV}_{\text {peak-peak }}$ and $25 \mathrm{kHz}$. We used a treatment distance of $5 \mathrm{~mm}$ between the end of the capillary tube and the surface of the bacterial biofilms ("contact mode"). The P. aeruginosa biofilms were all incubated for a total time of $24 \mathrm{~h}$, removed from incubation at varying time points $(0,4,8,12,20$, and $24 \mathrm{~h})$, and subjected to $5 \mathrm{~min}$ of plasma treatment before being reincubated for the remaining time (with the exception of the 24-h biofilms that were assessed immediately after treatment).

\section{E. Scanning Electron Microscopy}

Biofilms were fixed overnight in glutaraldehyde (1.5\%) and paraformaldehyde $(3 \%)$ in phosphate buffer ( $\mathrm{pH}$ 7.3). Samples were rinsed with osmium tetroxide and dehydrated in ethanol/water mixtures at increasing concentrations. Biofilms were sputter coated with gold and imaged via scanning electron microscope (SEM) JEOL SEM6480LV (Tokyo, Japan), operated at $10 \mathrm{kV}$.

Volume 8, Issue 3, 2018 


\section{F. Live/Dead Staining and Confocal Microscopy}

Biofilms were washed three times in PBS to remove planktonic bacteria. We prepared BacLight ${ }^{\mathrm{TM}}$ stains (consisting of two nucleic acid dyes of SYTO-9 and propidium iodide) according to manufacturer instructions. Each biofilm was immersed in $1.5 \mathrm{~mL}$ of stain mixture and incubated for $15 \mathrm{~min}$ in the dark. After staining, biofilms were rinsed once with PBS, fixed onto a microscope slide, and imaged using a confocal microscope to obtain Z-stacked images of the bacterial biofilms.

\section{RESULTS AND DISCUSSION}

The effect of plasma jet treatment of $P$. aeruginosa biofilms at varying time points during biofilm maturation is shown in Fig. 1. Relative to the untreated control, we found a significant reduction in the number of viable bacterial cells at each treatment intervention point, demonstrating a clear disruption in the formation of mature biofilms as a result of CAP exposure.

CAP treatment at 0 and $4 \mathrm{~h}$ produced a $5-\log$ reduction in CFU/mL, reducing bacterial load below the clinically relevant value of $10^{6} \mathrm{CFU} / \mathrm{mL} .^{23,24}$ However, CAP treatment at later stages during biofilm maturation $(12,20$, and $24 \mathrm{~h})$ reduced CFU $/ \mathrm{mL}$ values by only 1-2-log units. From these data, we notice a critical time frame for treatment intervention to limit bacterial proliferation within a biofilm. Although the exact

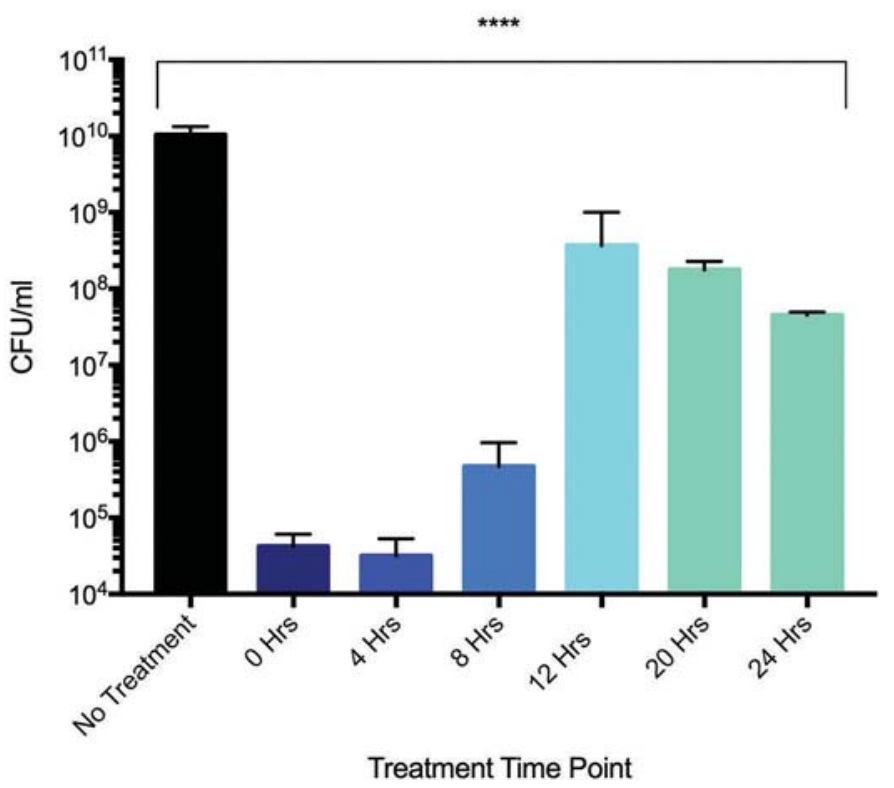

FIG. 1: Effect of treatment intervention time on bacterial viability after $24 \mathrm{~h}$ of incubation. CAP treatment was carried out as previously described at the time points shown $(p<0.0001$; one-way analysis of variance with multiple comparisons) 
reason for this is unclear at this point, a number of possible factors may play a part in the resistance of mature biofilms to plasma exposure, for example, EPS production and/or a change in bacterial genotype/phenotype within the biofilms. The difference in cell counts at the varying stages of intervention may indeed have a role in the susceptibility of bacteria to plasma treatment. However, owing to the fact that each biofilm is incubated for $24 \mathrm{~h}$ regardless of treatment time, the results suggest that not only does CAP treatment reduce the number of viable cells, it also prevents the recovery of biofilms into the mature state.

We carried out qualitative analysis of the biofilms before and after CAP treatment using SEM to look more closely and evaluate the effect of CAP exposure on a cellular level. Figure 2(A) shows an untreated $P$. aeruginosa biofilm grown for $24 \mathrm{~h}$. The bacterial cells are present in high density, reflecting the high CFU/ $\mathrm{mL}$ value calculated in the previous quantitative data (Fig. 1). The presence of the EPS matrix is clearly visible, holding the cells in close proximity to one another. Figure 2(B) shows a $P$. aeruginosa biofilm incubated for $24 \mathrm{~h}$ but treated with
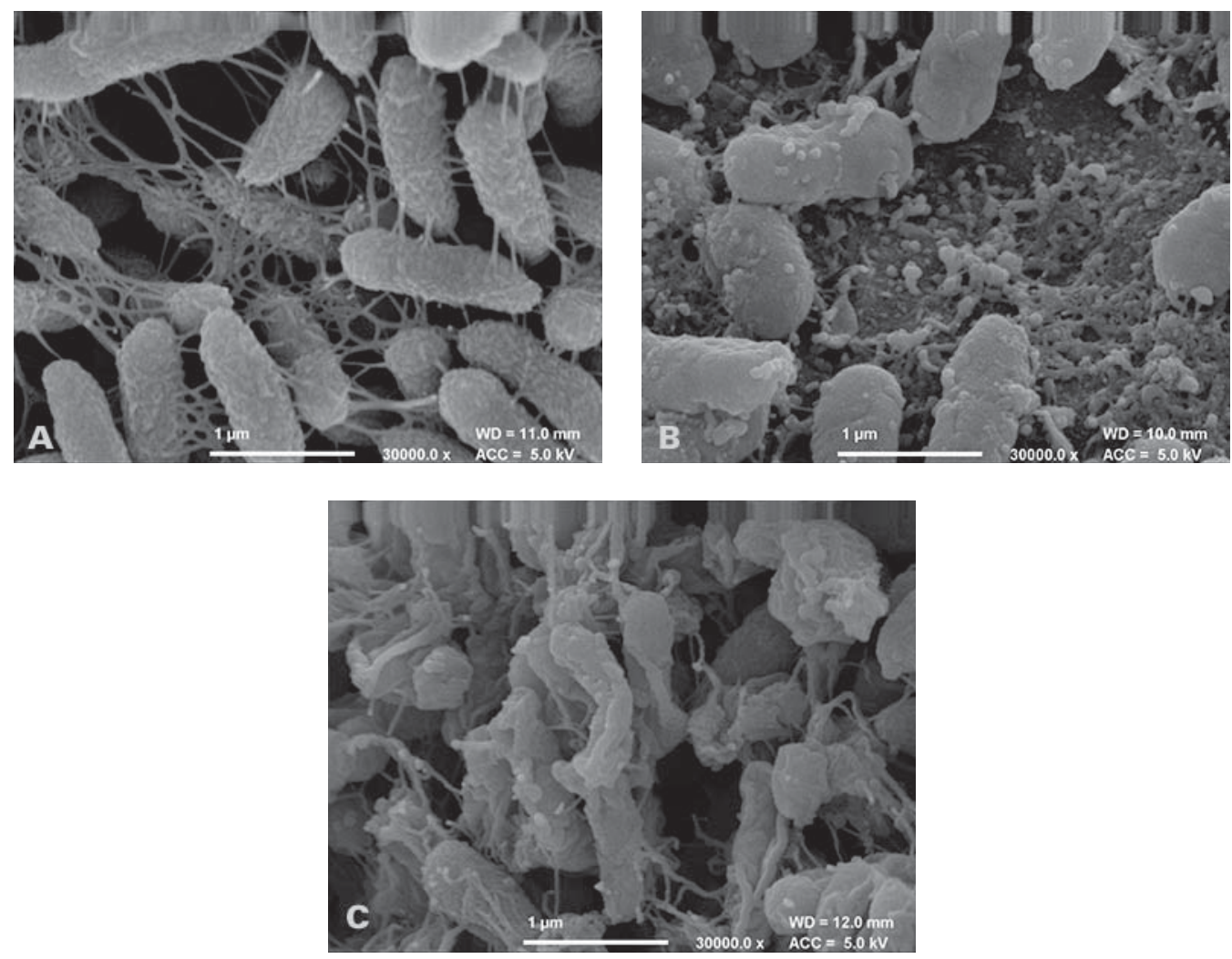

FIG. 2: SEM images of CAP-treated biofilms. (A) Untreated 24-h growth P. aeruginosa biofilm (control); (B) 24-h P. aeruginosa biofilm treated with CAP jet for 5 min at 8-h growth; (C) 24-h $P$. aeruginosa biofilm treated with CAP jet for $5 \mathrm{~min}$ at 12 -h growth

Volume 8, Issue 3, 2018 
CAP jet at $8 \mathrm{~h}$ of growth. Relative to Fig. 2(A), this shows a distinct reduction in bacterial cell density alongside an accumulation of cellular debris, likely the result of bacterial cell death during CAP treatment. There is also a clear reduction in the EPS matrix. Figure 2(C) shows a $P$. aeruginosa biofilm grown for $24 \mathrm{~h}$, treated with the CAP jet at $12 \mathrm{~h}$ of growth. As expected from the quantitative data, we see a higher density of cells relative to the biofilms treated at $8 \mathrm{~h}$. However, cell morphology suggests significant bacterial cell death and a clear disruption to the EPS relative to the untreated control [Fig. 2(A)]. Interestingly, despite the higher number of viable cells when treating the biofilms at $12 \mathrm{~h}$ relative to $8 \mathrm{~h}$, the ability of the former to recover to full cell density as expected in a mature biofilm is reduced, potentially reflecting the disruption in both bacterial cells and EPS matrix, as shown in Fig. 2(C).

To further investigate the 3-log difference in CFU/mL between biofilms treated at 8 and $12 \mathrm{~h}$, we carried out live/dead staining to assess difference in viable bacteria. Figure 3 shows the difference in cell density between biofilms treated at the two different intervention points. As expected from the previous quantitative and qualitative data, a significantly higher density of cells can be seen in the biofilms treated at 12 h [Fig. 3(B)]. Figure 3(A) shows a thin layer of healthy viable cells, likely the result of the 16-h post-treatment recovery period to which the biofilm was subjected, supporting the presentation of healthy cells in Fig. 2(B). Early treatment of the biofilms ( $8 \mathrm{~h}$ and less) provides adequate time for the recovery of viable bacterial cells (albeit not to the full cell density seen in untreated, mature biofilms during the same time period). However, in Fig. 3(B), we clearly find a larger proportion of dead bacteria, suggested by SEM [Fig. 2(C)]. The density of the bacterial biofilm provides an impenetrable layer of biological material that protects cells in the lower levels of the biofilm from the plasma jet action. Despite the ability of CAP treatment to cause significant cellular lysis, the protective nature of the more established biofilms shields the cells in the lower layers of the biofilm, thus retaining cell viability (Fig. 1) despite the presence of dead cells (Figs. 2 and 3).

\section{CONCLUSIONS}

Using CAP therapy in a time-dependent manner is crucial for reducing the formation of mature $P$. aeruginosa biofilms. Although CAP therapy is able to cause significant bacterial cell death, the presence of both dead and living cells contained within an established biofilm offers protective effects relative to the cells in the lower layers of the biofilm, resulting in retention of viable cells. However, treating biofilms in the early stages of development $(<12 \mathrm{~h})$ can significantly reduce bacterial loads to levels, wherein traditional treatment strategies may become effective. Using CAP therapy as a tool to limit biofilm formation may prove to be clinically advantageous by increasing the potential for immune system clearance without the need for pharmaceutical intervention. Furthermore, CAP treatment could be effectively used in tandem with antibiotics by disrupting biofilm formation, thus reducing the concentration of antimicrobial required. This technology 

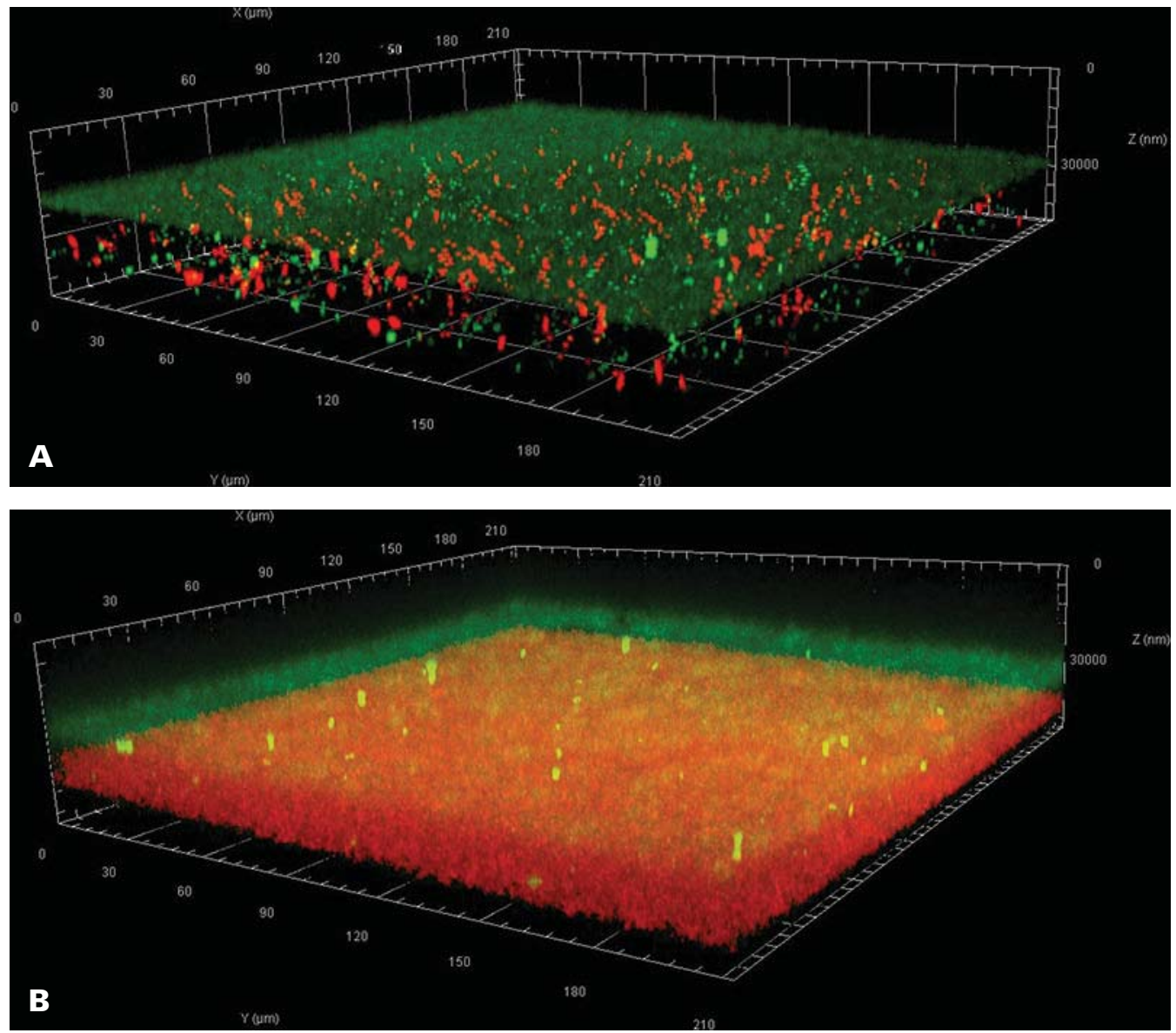

FIG. 3: BacLight ${ }^{\mathrm{TM}}$ live/dead staining of $24 \mathrm{~h}$ P. aeruginosa biofilms. (A) Treated with CAP jet for 5 min at $8 \mathrm{~h}$ growth; (B) treated with CAP jet for 5 min at $12 \mathrm{~h}$ growth. SYTO9 stains all cells Light Grey (LIVE) and PI only stains cells with damaged cytoplasmic membrane Dark Grey (DEAD). Images are inverted, representing the biofilms from the base of the membrane downwards.

therefore has the capacity to contribute to the global aim of decreased reliance on antibiotic use.

\section{ACKNOWLEDGMENTS}

The authors thank the Engineering and Physical Sciences Research Council (EPSRC) for Grant No. EP/R003556/1. B.L.P. thanks James Tudor and Alastair and Nathalie Watson for additional funding. G.T.W. is grateful to the EPSRC and Public Health England. A.T.A.J., A.C.S., and N.T.T. thank the EPSRC for funding smart-wound plasma (Grant 
No. EP/R003939/1). We are grateful to the Microscopy and Analysis suite at the University of Bath, UK for assistance with SEM and confocal microscopy.

\section{REFERENCES}

1. Flynn PB, Higginbotham S, Alshraiedeh NaH, Gorman SP, Graham WG, Gilmore BF. Bactericidal efficacy of atmospheric pressure non-thermal plasma (APNTP) against the ESKAPE pathogens. Int J Antimicrob Agents. 2015;46(1):101-7.

2. Modic M, McLeod NP, Sutton JM, Walsh JL. Cold atmospheric pressure plasma elimination of clinically important single- and mixed-species biofilms. Int J Antimicrob Agents. 2017;49(3):375-8.

3. Fridman G, Friedman G, Alexander G, Shekhter A, Anatoly SB. Vasilets VN, Fridman A. Applied Plasma Medicine. Plasma Proc Polymers. 2008;5(6):503-33.

4. Alkawareek MY, Algwari QT, Laverty G, Gorman SP, Graham WG, O’Connell D, Gilmore BF. Eradication of Pseudomonas aeruginosa biofilms by atmospheric pressure non-thermal plasma. PLoS ONE. 2012;7(8):e44289.

5. Boxhammer V, Morfill GE, Jokipii JR, Shimizu T, Klämpfl T, Li Y-F, Köritzer J, Schlegel J, Zimmermann JL. Bactericidal action of cold atmospheric plasma in solution. New J Phys. 2012;14(11) 113042.

6. Gilmore BF, Flynn PB, O’Brien S, Hickok N, Freeman T, Bourke P. Cold plasmas for biofilm control: Opportunities and challenges. Trends Biotechnol. 2018;36(6):627-38.

7. Padrig BF, Brendan FG. Understanding plasma biofilm interactions for controlling infection and virulence. J Phys D Appl Phys. 2018;51(26):263001.

8. Haertel B, von Woedtke T, Weltmann K-D, Lindequist U. Non-thermal atmospheric-pressure plasma possible application in wound healing. Biomol Therapeut. 2014;22(6):477-90.

9. Mai-Prochnow A, Murphy AB, McLean KM, Kong MG, Ostrikov K. Atmospheric pressure plasmas: Infection control and bacterial responses. Int J Antimicrob Agents. 2014;43(6):508-17.

10. O'Connor N, Cahill O, Daniels S, Galvin S, Humphreys H. Cold atmospheric pressure plasma and decontamination. Can it contribute to preventing hospital-acquired infections? J Hosp Infect. 2014;88(2):59-65.

11. Xu GM, Shi XM, Cai JF, Chen SL, Li P, Yao CW, Chang ZS, Zhang GJ. Dual effects of atmospheric pressure plasma jet on skin wound healing of mice. Wound Repair Regen. 2015;23(6):878-84.

12. von Woedtke T, Metelmann H-R, Weltmann K-D. Clinical plasma medicine: State and perspectives of in vivo application of cold atmospheric plasma. Contrib Plasma Phys. 2014;54(2):104-17.

13. Percival SL, McCarty SM, Lipsky B. Biofilms and wounds: An overview of the evidence. Adv Wound Care (New Rochelle). 2015;4(7):373-81.

14. Olson ME, Ceri H, Morck DW, Buret AG, Read RR. Biofilm bacteria: Formation and comparative susceptibility to antibiotics. Can J Vet Res. 2002;66(2):86-92.

15. Stewart PS. Diffusion in biofilms. J Bacteriol. 2003;185(5):1485-91.

16. Heurlier K, Dénervaud V, Haas D. Impact of quorum sensing on fitness of Pseudomonas aeruginosa. Int J Med Microbiol. 2006;296(2):93-102.

17. Ceri H, Olson ME, Stremick C, Read RR, Morck D, Buret A. The Calgary biofilm device: New technology for rapid determination of antibiotic susceptibilities of bacterial biofilms. J Clin Microbiol. 1999;37(6):1771-6.

18. Morrison AJ, Wenzel RP. Epidemiology of infections due to Pseudomonas aeruginosa. Rev Infect Dis. 1984;6(Suppl. 3):S627-42.

19. Gellatly SL, Hancock REW. Pseudomonas aeruginosa: New insights into pathogenesis and host defenses. Pathogens Dis. 2013;67(3):159-73.

20. Livermore DM. Multiple mechanisms of antimicrobial resistance in Pseudomonas aeruginosa: Our worst nightmare? Clin Infect Diseases. 2002;34(5):634-40.

21. Thet NT, Wallace L, Wibaux A, Jenkins ATA. Development of a mixed-species biofilm model and its virulence implications in device related infections. J Biomed Mater Res Appl Biomater. 2018 Mar 8. doi: $10.1002 / \mathrm{jbm}$. b.34103. 
22. Szili EJ, Gaur N, Hong SH, Kurita H, Oh JS, Ito M, Mizuno A, Hatta A, Cowin AJ, Graves DB, Short RD. The assessment of cold atmospheric plasma treatment of DNA in synthetic models of tissue fluid, tissue and cells. J Phys D Appl Phys. 2017;50(27):274001-1-15.

23. Jacobs MR, Lazarus HM, Yomtovian RA. Relationship between bacterial load, species virulence, and transfusion reaction with transfusion of bacterially contaminated platelets. Clin Infect Dis. 2008;46(8):1214-20.

24. Opota O, Croxatto A, Prod'hom G, Greub G. Blood culture-based diagnosis of bacteraemia: State of the art. Clin Microbiol Infect. 2015;21(4):313-22.

Volume 8, Issue 3, 2018 
